\section{Bronchial rupture secondary to blunt chest trauma}

\author{
C Hartley, G N Morritt
}

\begin{abstract}
Bronchial injury due to blunt chest trauma is rare, and its rarity and the fact that it has two distinct modes of presentation may considerably delay diagnosis. Two recent cases illustrate the two main types of injury and presentation. In the first the rupture is intrapleural and air escapes into the pleural space; insertion of a chest drain leads to a continuous air leak. In the second type the rupture is largely extrapleural with little communication with the pleural cavity; initially symptoms may be mild or absent but complications may occur later.
\end{abstract}

(Thorax 1993;48:183-184)

External penetrating injury and endoscopic and intubation accidents are well recognised as a cause of bronchial rupture and should arouse a high index of suspicion that this may be the diagnosis. Cases due to blunt trauma represent a more difficult diagnostic problem. Two recent cases illustrate how the presentation may vary and diagnostic difficulty arise.

\section{Case reports}

PATIENT 1

A 17 year old motorcyclist was admitted after a road traffic accident. On arrival in the accident and emergency department he was semiconscious and cyanosed and had gross surgical emphysema. A chest radiograph showed a large right and smaller left pneumothorax. A right sided intercostal drain was inserted, with a resultant large air leak through the tube. A second right and also a left chest drain were therefore inserted. Other injuries included fractures of the right clavicle, scapula, and first and second ribs. A provisional diagnosis of rupture of a bronchus was confirmed by bronchoscopy. At thoracotomy a complete circumferential tear of the right main bronchus was found $1.5 \mathrm{~cm}$ from the carina. The right main bronchus was anastomosed, the suture line being reinforced with an intercostal pedicel graft. The patient therefter made an unremarkable recovery.

PATIENT 2

A 14 year old boy was admitted after a 120 foot fall from a railway bridge. He was noted to have a right tension pneumothorax with associated mediastinal and surgical emphysema. This was managed successfully by inserting an intercostal tube and he was taken to theatre for repair of a ruptured liver. Other injuries included fractures of the right maxilla, scapula, and pelvis. Eight days after the injury a chest radiograph showed collapse of the right middle and lower lobes. Fibreoptic bronchoscopy showed "an odd membranous type partial stenosis" of the right main bronchus. The radiographic abnormality resolved after bronchoscopy. Twenty five days after injury he developed increasing dyspnoea and stridor and further bronchoscopy showed almost complete stenosis of the right main bronchus. When he was transferred to the regional cardiothoracic unit his forced expiratory volume in one second $\left(\mathrm{FEV}_{1}\right)$ was 1.4 litres and vital capacity (VC) 1.81 . He had two bronchoscopies, during which the YAG laser was used to burn away tissue obstructing the bronchial lumen. This was followed by bougie dilatation to size 24 (French gauge), and his $F E V_{1}$ rose to 2.41 and his VC to 2.951 . Within two weeks, however, his lung function had again deteriorated, and this was reflected in increasing dyspnoea and stridor. Bronchoscopy showed only a $2 \mathrm{~mm}$ lumen in the right main bronchus. Bronchography was undertaken to clarify the extent of the stricture and its location in relation to the orifice of the right upper lobe bronchus (fig 1).

Exactly four months after injury the patient underwent a thoracotomy with resection of the stricture and anastomosis of the right main bronchial stump to the carina. He was symptom free with excellent lung function on review in the clinic one month later.

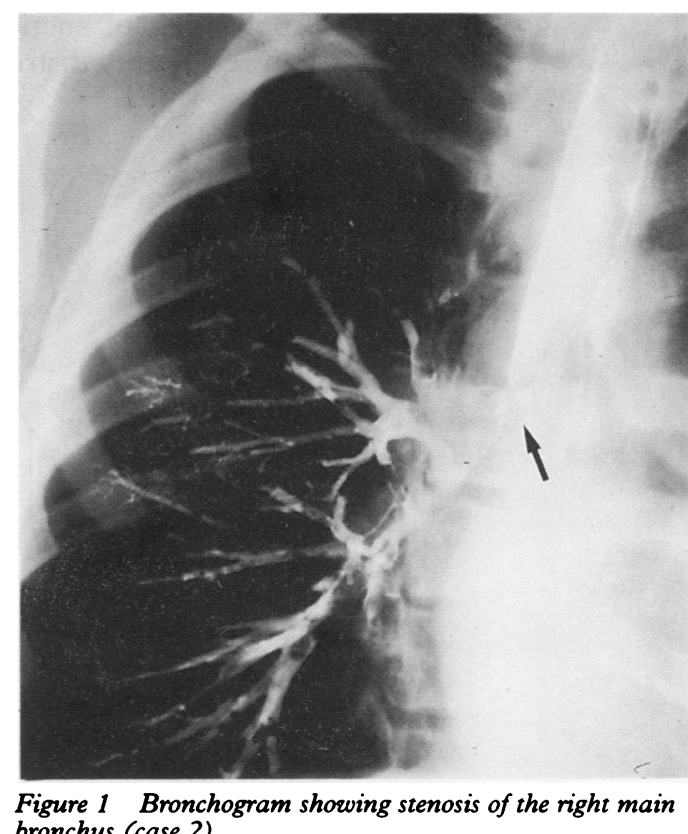

Figure 1 Bronchogram showing stenosis of the right main bronchus (case 2).
Received 6 February 1992 Returned to auth Revised version received
Reviscis 1992 28 April 1992

Accepted 7 May 1992 


\section{Discussion}

The incidence of bronchial rupture is low $(0.03-2.5 \%)$ even in those with severe trauma, ${ }^{1-3}$ though it increased as the number of road traffic accidents rose. It is estimated that a thoracic surgeon in the United Kingdom would see only two such cases, one acute, in 30 years. ${ }^{4}$ Most bronchial injuries occur in males under 40 years of age-a male:female ratio of $3: 1 .^{1}$

Of patients suffering a bronchial injury, a large proportion $(30-80 \%)$ will die before reaching hospital, mainly of associated injuries. ${ }^{5}$ Most deaths occur within one hour of injury. ${ }^{56}$ Patients with bronchial rupture reaching hospital alive have a $90 \%$ chance of survival $^{6}$ and half of such patients will have no other injury. ${ }^{1}$ Chest wall injuries occur in a third of survivors and might be more common but for the compliant nature of the chest in young adults. ${ }^{1}$ In patients over 30 years of age fracture of one of the upper three ribs strongly suggests bronchial rupture. ${ }^{15}$

Most bronchial injuries can be accounted for by three mechanisms ${ }^{17}$ : firstly, compression of the chest between sternum and vertebral column; secondly, a sudden deceleration of the pendulous lung, producing a shearing force at the fixed trachea; and, thirdly, forced expiration from a position of full inspiration against a fixed glottis (that is, "bracing oneself"), which may cause a blow out of the membranous portion of the trachea.

Eighty per cent of bronchial ruptures occur within $2.5 \mathrm{~cm}$ of the carina. ${ }^{178}$ Injuries are equally distributed between left and right main bronchi, though rarely more distal airways are affected. ${ }^{8}$

Patient 1 illustrates the more obvious presentation. The rupture is intapleural, air escaping freely into the pleural space. This has been termed a type 1 injury9 (figure 2). Radiographically, a large pneumothorax is present, with variable mediastinal emphysema. The "fallen lung sign" may be present. ${ }^{6}$ Inserting a chest drain leads to a continuous air leak, and the lung fails to re-expand. ${ }^{9}$ Application of suction increases the air leak and respiratory distress. ${ }^{8}$

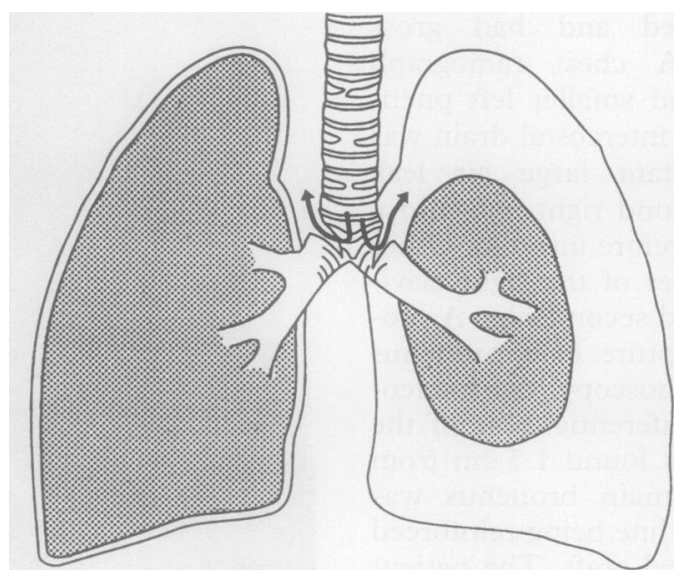

Figure 2 Diagrammatic representation of sites of rupture and the consequent air leak: type 1 rupture illustrated by $a$ tear of the left main bronchus; type 2 rupture illustrated by a tear of the right main bronchus.
Patient 2 illustrates a type 2 injury with little communication between the largely extrapleural tear and the pleural cavity. ${ }^{9}$ If a pneumothorax is present it readily improves with insertion of an intercostal drain. ${ }^{16}$ Patients may have relatively mild respiratory symptoms or be symptom free $(10 \%) .{ }^{16}$ Haemoptysis from torn bronchial vessels may occur, but is more commonly absent. Later there is repair of the bronchial tear by granulation tissue with possible stricture formation and distal collapse. Cough, dyspnoea, or stridor may occur weeks, months, or even years later. ${ }^{9}$ The distal lung may be affected by recurrent infections or bronchiectasis. ${ }^{69}$ If delayed collapse of the lung occurs it will do so within two months of the injury. ${ }^{1}$ Once suspected, the diagnosis is usually confirmed by bronchoscopy, though bronchography, tomography and computed tomography have all been used. ${ }^{510}$ Although bronchoscopy in the early stages may not prove diagnostic it remains the investigation of choice and should be repeated if diagnostic uncertainty remains. ${ }^{6}$ Only a third of cases are diagnosed within the first 24 hours, ${ }^{10} 40 \%$ being diagnosed more than one month after injury. ${ }^{1}$

Surgical repair of a ruptured bronchus was first performed successfully in 1948 and is now the treatment of choice. ${ }^{2}$ Primary repair results in preservation of lung function and good long term results in over $90 \%$ of patients. ${ }^{6}$ It may not be possible owing to the position or extent of the tear. Lobectomy or pneumonectomy is sometimes necessary. ${ }^{6}$

Where a stricture has occurred treatment depends on the condition of the distal lung. ${ }^{6}$ If the stenosis is complete the distal lung will be healthy ${ }^{16}$ and secondary repair is possible in three quarters of cases, being a good means of restoring lung function, though not always to normal levels. ${ }^{16}$ If the stenosis is partial the distal lung is more likely to be affected by recurrent infections or bronchiectasis. ${ }^{610}$ Thus lobectomy (in one out of three cases) or pneumonectomy (two out of three) may be required. ${ }^{6}$ Both primary and secondary repair may be followed by recurrence of stenosis in a few cases but this complication, unlike the original stenosis, responds to dilatation. ${ }^{67}$

1 Chesterman JT, Satsangi PN. Rupture of the trachea and bronchi by closed injury. Thorax 1966;21:21-7.

2 Griffith JL. Fracture of the bronchus. Thorax 1949;4: 105-9.

3 Alonyo D, Perry JF Jr. Impact of speed limit-a review of 966 cases. I Thorac Cardiovasc Surg 1982;83:519-22.

4 Bates M. Rupture of the bronchus. In: Williams WG, Smith RE, eds. Trauma of the chest Bristol: Wright, 1977:142-50.

5 Burke JF. Early diagnosis of traumatic rupture of the bronchus. $¥ A M A$ 1962;181:682-6.

6 Roxburgh JC. Rupture of the tracheobronchial tree. Thorax 1987;42:681-8.

7 Amauchi W, Birolini D, Branco PD, De Olivera MR. Injuries to the tracheobronchial tree in closed trauma. Thorax 1983;38:923-8.

8 Kirsh MM, Oringer MB, Behrendt DM, Sloan $H$. Management of tracheobronchial disruption secondary to nonpenetrating trauma. Ann Thorac Surg 1976;22: 93-101.

9 Davies D, Hopkins JS. Patterns in traumatic rupture of the bronchus. Injury 1972-3;4:261-4.

10 Deslauriers J< Beaulieu M, Archambault G, LaForge J, Bernier R. Diagnosis and long-term follow-up of major bronchial disruptions due to nonpenetrating trauma. Ann Thorac Surg 1982;33:32-9. 